Research Article

\title{
Error Modeling and Sensitivity Analysis of a Five-Axis Machine Tool
}

\author{
Wenjie Tian, ${ }^{1}$ Weiguo Gao, ${ }^{1}$ Wenfen Chang, ${ }^{1,2}$ and Yingxin $\mathrm{Nie}^{1,2}$ \\ ${ }^{1}$ Key Laboratory of Mechanism Theory and Equipment Design of Ministry of Education, Tianjin University, Tianjin 300072, China \\ ${ }^{2}$ Beijing Precision Machinery \& Engineering Research Co., Ltd., Beijing 101312, China
}

Correspondence should be addressed to Weiguo Gao; gaowg@tju.edu.cn

Received 20 October 2013; Revised 27 February 2014; Accepted 27 February 2014; Published 29 May 2014

Academic Editor: Miguel A. F. Sanjuán

Copyright (C) 2014 Wenjie Tian et al. This is an open access article distributed under the Creative Commons Attribution License, which permits unrestricted use, distribution, and reproduction in any medium, provided the original work is properly cited.

Geometric error modeling and its sensitivity analysis are carried out in this paper, which is helpful for precision design of machine tools. Screw theory and rigid body kinematics are used to establish the error model of an RRTTT-type five-axis machine tool, which enables the source errors affecting the compensable and uncompensable pose accuracy of the machine tool to be explicitly separated, thereby providing designers and/or field engineers with an informative guideline for the accuracy improvement by suitable measures, that is, component tolerancing in design, manufacturing, and assembly processes, and error compensation. The sensitivity analysis method is proposed, and the sensitivities of compensable and uncompensable pose accuracies are analyzed. The analysis results will be used for the precision design of the machine tool.

\section{Introduction}

Geometric accuracy is a crucially important performance factor for machine tools, especially under circumstances where relatively high precision is one of the basic requirements [1]. There are two ways to improve the geometric accuracy of machine tools: (1) design and manufacture for precision and (2) error compensation. Both require a parametric model that relates the geometric source errors to the pose accuracy of the cutting tool relative to the workpiece. Theoretically, the effects of source errors on pose accuracy of 3-, 4-, or 5-axis machine tools cannot fully be compensated by software, and only those pose errors corresponding to the permissible motion types are compensable by means of error compensation. Therefore, a comprehensive error model is essential in order to distinguish the source errors affecting compensable pose errors from those affecting uncompensable ones. Only then can suitable measures be adopted for accuracy improvement $[2,3]$ via component tolerance design, manufacturing, and assembly, as well as error compensation.

Of all types of source errors, geometric source errors have the largest influences on the workpiece accuracy, which should be considered first in design. Over the past decades, there has been a great deal of intensive research into error modeling of machine tools. Homogeneous transformation matrix (HTM) or Denavit-Hartenberg (D-H) transformation matrix is widely used to describe the rigid body kinematics. Kiridena and Ferreira [4] classified 5-axis machine tools into TTTRR, RTTTR, and RRTTT systems and used the D-H convention to develop kinematic models for each of these three machine types. However, their model considered only five parametric errors (one positioning error for each axis). Srivastava et al. [5] established a combined error model, which considered both geometric errors and thermal errors. For error compensation of a 5-axis machine tool, Jha and Kumar. [6] established the volumetric error model with D-H method. Lin and Shen [7] proposed a new matrix summation method; the kinematic equation was converted into six components to give each component a clear physical meaning. However, the physical meaning of geometric source error was not clear. Yang [8] dedicated one chapter of his dissertation to the formulation of a generalized $5 \mathrm{D}$ error synthesis model. However, he defined only 27 geometric error components, which is incomplete. Similar work has been done for the purpose of error modeling of machine tools [9-14]. In the past decades, most studies mainly aimed at error compensation, and little attention has been paid to the important design question of separating the source 
errors into two subsets associated with the compensable and uncompensable pose accuracy.

Concerning the error sensitivity analysis, Huang et al. [15] established a statistical sensitivity model and analyzed the sensitivity of uncompensable pose accuracy with respect to the geometric source errors for a 3-DOF parallel kinematic machine with parallelogram struts. Chanal et al. [16] and Fan et al. [17] did the sensitivity analysis of another two PKMs with similar method. And many similar work [18, 19] has been done. Chen et al. [20] analyzed the sensitivity of the volumetric error regarding 37 source error components for designing a five-axis machine tool. However, only three special configurations were chosen to perform the analysis, which were probably insufficient.

In this paper, after Section 1 has briefly addressed current challenges in error modeling and sensitivity analysis of machine tools, Section 2 introduces two kinds of geometric source errors within the machine tools. In Section 3, a systematic model is established, which formulates the linear map between the pose error twist and geometric source errors within a machine tool using the homogeneous transformation matrix method. Section 4 gives an error model that fully clarifies which source errors affect the compensable and uncompensable pose accuracy. Finally, Section 5 proposes a new method for sensitivity analysis of multiaxis machine tools and takes a typical five-axis machine tool as an example to illustrate the generality and effectiveness of this approach before conclusions are drawn in Section 6.

\section{Geometric Source Errors of a Five-Axis Machine Tool}

2.1. Machine Tool Structure. The schematic diagram of a fiveaxis machine tool (RRTTT-type) is shown in Figure 1. The machine tool has three translational axes and two rotational axes, that is, $X$-axis, $Y$-axis, $Z$-axis, $A$-axis, and $C$-axis, respectively. The tool system is supported by the $Z$-axis and the workpiece is supported by the $C$-axis. The $C$-axis is supported by the $A-, X$ - and $Y$-axis sequentially.

2.2. Geometric Source Errors. According to the definitions proposed in [20], the geometric error of the machine tool refers to the error of individual axes and those between axes, such as linear/angular positioning errors, straightness errors, orientational errors, offset errors, and squareness errors. Every translational/rotational axis has six positiondependent geometric source errors. All position-dependent errors of this machine tool are shown in Table 1. Positionindependent errors of a machine tool refer to the location errors of an axis from its nominal pose [20]. In this paper, we use $S_{x y}$ to express the squareness error between $X$-axis and $Y$-axis. For the machine tool shown in Figure 1, with the method presented in [20], the real motion direction of $Y$-axis is selected as the reference direction; so $Y$-axis has no location errors. The plane through $X$-axis and $Y$-axis is selected as the reference plane; so $X$-axis has one squareness error $S_{x y}$, and $Z$-axis has two squareness errors $S_{x z}$ and $S_{y z}$. Similarly, $A$ axis has two squareness errors $S_{\alpha z}$ and $S_{\alpha z}$, and $C$-axis has two squareness errors $S_{\gamma x}$ and $S_{\gamma y} . \delta_{x A}, \delta_{y A}$, and $\delta_{z A}$ are the

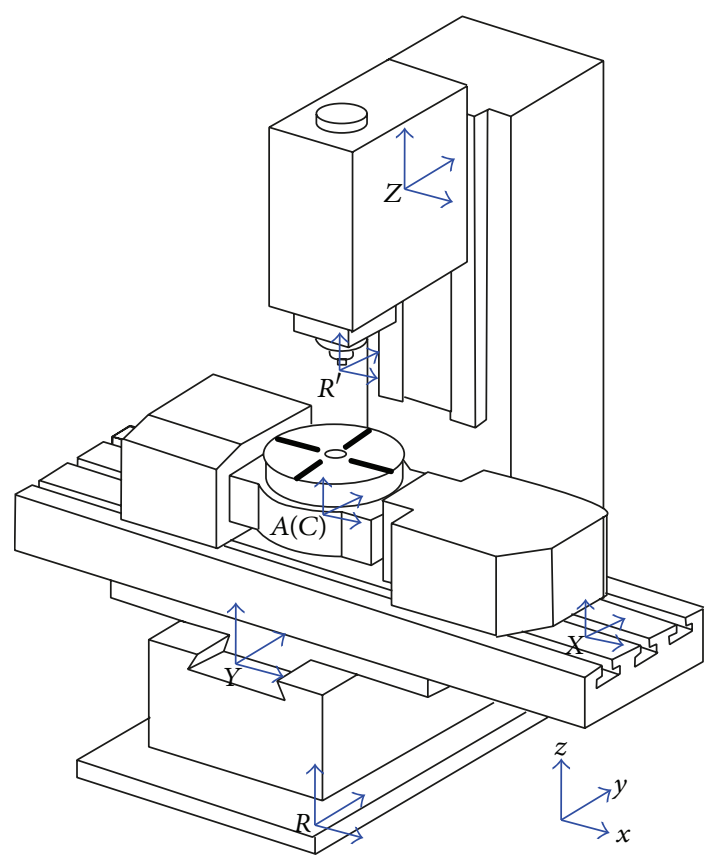

FIgURE 1: Schematic diagram of a 5-axis machine tool.

linear offset errors of $A$-axis with respect to $X$-axis in $X, Y$, and $Z$ directions, respectively. $\delta_{y C}$ is the offset error of the $C$ axis with respect to $A$-axis in $Y$ direction, since the two rotary axes may not intersect perfectly.

\section{Error Modeling}

Error modeling aims to establish a map from geometric source errors to the pose errors of machine tool, which is the common premise of precision design and error compensation. All kinds of machine tools have two structural chains which contain several components connected in series by prismatic and rotational joints. One is from machine bed to workpiece, and the other is from machine bed to cutting tool, which are represented by workpiece kinematic chain and cutting tool kinematic chain, respectively. Thus, establishing the error model of kinematic chain is the foundation of the whole machine tool error model.

3.1. Error Modeling for Workpiece Kinematic Chain. Figure 2(a) shows the workpiece kinematic chain of the five-axis machine tool. This chain has four joints, two translational joints and two rotational joints, and, here, we use $i=1,2,3,4$ to present the $Y-, X-, A-$, and $C$-axis sequentially. Modeling the chain error uses two global reference frames $R$ and $R^{\prime}: R$ is located at point $O$ on the machine bed, while $R^{\prime}$ is located at point $O^{\prime}$ on the workpiece and remains parallel to $R$. In order to describe the source errors within the chain and relate them to pose accuracy of workpiece, body fixed frame $R_{W}$ is placed at point $O^{\prime}$, and body fixed frames $R_{i}$ are placed on the $i$ th joint ( $i=1, \ldots, 4)$. As previously mentioned, the source errors can be divided into two categories: (1) position-dependent 
TABLE 1: Geometric source errors of 5-axis machine tools.

\begin{tabular}{llr}
\hline Axis & Position-dependent errors & Position-independent errors \\
\hline$X$-axis & $\delta_{x}(x), \delta_{y}(x), \delta_{z}(x), \varepsilon_{x}(x), \varepsilon_{y}(x), \varepsilon_{z}(x)$ & $S_{x y}$ \\
$Y$-axis & $\delta_{x}(y), \delta_{y}(y), \delta_{z}(y), \varepsilon_{x}(y), \varepsilon_{y}(y), \varepsilon_{z}(y)$ & $S_{z y}, S_{z x}$ \\
$Z$-axis & $\delta_{x}(z), \delta_{y}(z), \delta_{z}(z), \varepsilon_{x}(z), \varepsilon_{y}(z), \varepsilon_{z}(z)$ & $\delta_{x A}, \delta_{y A}, \delta_{z A}, S_{\alpha z}, S_{\alpha y}$ \\
$A$-axis & $\delta_{x}(\alpha), \delta_{y}(\alpha), \delta_{z}(\alpha), \varepsilon_{x}(\alpha), \varepsilon_{y}(\alpha), \varepsilon_{z}(\alpha)$ & $\delta_{y C}, S_{\gamma y}, S_{\gamma x}$ \\
$C$-axis & $\delta_{x}(\gamma), \delta_{y}(\gamma), \delta_{z}(\gamma), \varepsilon_{x}(\gamma), \varepsilon_{y}(\gamma), \varepsilon_{z}(\gamma)$ & \\
\hline
\end{tabular}

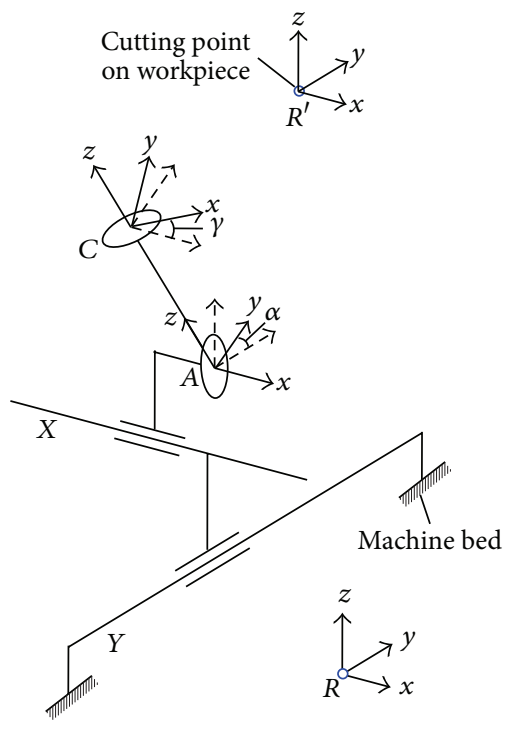

(a)

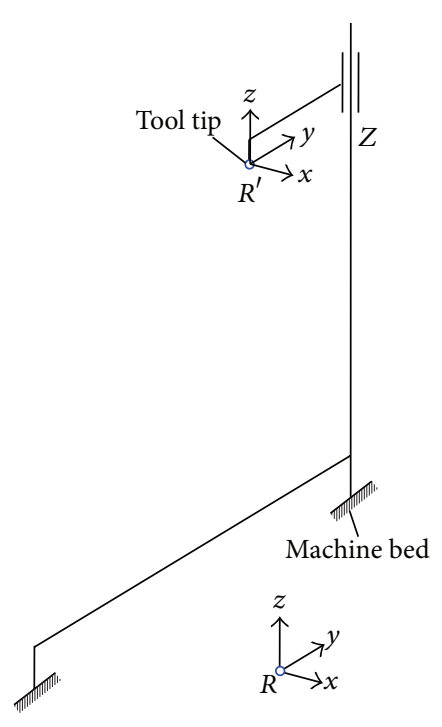

(b)

FIGURE 2: Two kinematic chains of the five-axis machine tool: (a) workpiece kinematic chain; (b) cutting tool kinematic chain.

errors, that is, the location errors of $R_{i}$ relative to $R_{i-1}$, and (2) position-independent errors, that is, the joint motion errors along/about axes measured in $R_{i}$.

At the nominal configuration, the pose (position and orientation) of the workpiece measured in $R$ can be expressed by successive $4 \times 4$ homogeneous transformations. Consider

$$
\begin{gathered}
{ }^{0} \mathbf{T}_{f+1}={ }^{0} \mathbf{T}_{1}{ }^{1} \mathbf{T}_{2} \ldots{ }^{i-1} \mathbf{T}_{i} \ldots{ }^{f-1} \mathbf{T}_{f}{ }^{f} \mathbf{T}_{f+1} \\
{ }^{i-1} \mathbf{T}_{i}=\left[\begin{array}{cc}
{ }^{i-1} \mathbf{R}_{i} & { }^{i-1} \mathbf{p}_{i} \\
\mathbf{0} & 1
\end{array}\right],
\end{gathered}
$$

where ${ }^{i-1} \mathbf{T}_{i}$ is the homogeneous transformation matrix of $R_{i}$, with respect to $R_{i-1},{ }^{i-1} \mathbf{p}_{i}$ is the position vector of the origin of $R_{i}$, and ${ }^{i-1} \mathbf{R}_{i}$ is its orientation matrix.

Consider initially that the only location errors are of $R_{i}$ relative to $R_{i-1}$ and the only joint motion errors are of the $i$ th joint. The actual workpiece pose measured in $R$ is then approximately expressed by

$$
{ }^{0} \mathbf{T}_{W}\left(\mathbf{I}_{4}+{ }^{W} \widehat{\Delta}_{W}\right)={ }^{0} \mathbf{T}_{i-1}\left(\mathbf{I}_{4}+\widehat{\boldsymbol{\Theta}}_{i}\right){ }^{i-1} \mathbf{T}_{i}\left(\mathbf{I}_{4}+\widehat{\Delta}_{i}\right){ }^{i} \mathbf{T}_{W},
$$

where $\mathbf{I}_{4}$ is the fourth-order identity matrix, ${ }^{W} \widehat{\Delta}_{W}$ denotes the pose error matrix of the workpiece measured in $R_{W}$, and $\widehat{\boldsymbol{\Theta}}_{i}$ is the location error matrix of $R_{i}$ relative to $R_{i-1}$ expressed by

$$
\begin{aligned}
& \widehat{\boldsymbol{\Theta}}_{i}=\left[\begin{array}{cc}
{\left[\delta \boldsymbol{\theta}_{i} \times\right]_{3 \times 3}} & \delta \mathbf{r}_{i} \\
\mathbf{0}_{1 \times 3} & 0
\end{array}\right]_{4 \times 4} \\
& \delta \boldsymbol{\theta}_{i}=\left(\begin{array}{lll}
\varepsilon_{x, i} & \varepsilon_{y, i} & \varepsilon_{z, i}
\end{array}\right)^{T}, \\
& \delta \mathbf{r}_{i}=\left(\begin{array}{lll}
\delta_{x, i} & \delta_{y, i} & \delta_{z, i}
\end{array}\right)^{T} .
\end{aligned}
$$

Here, $\delta_{x, i}\left(\varepsilon_{x, i}\right), \delta_{y, i}\left(\varepsilon_{y, i}\right)$, and $\delta_{z, i}\left(\varepsilon_{z, i}\right)$ are the location error components of $R_{i}$ relative to $R_{i-1}$ along (about) three orthogonal axes of $R_{i-1},\left[\delta \boldsymbol{\theta}_{i} \times\right]$ is the skew-symmetric matrix of vector $\delta \boldsymbol{\theta}_{i}$ relating to its vector product, and $\widehat{\boldsymbol{\Delta}}_{i}$ is the joint motion error matrix measured in $R_{i}$ and has the form

$$
\widehat{\boldsymbol{\Delta}}_{i}=\left[\begin{array}{cc}
{\left[\delta \boldsymbol{\theta}_{i}\left(q_{i}\right) \times\right]_{3 \times 3}} & \delta \mathbf{r}_{i}\left(q_{i}\right) \\
\mathbf{0}_{1 \times 3} & 0
\end{array}\right]_{4 \times 4} .
$$

Here, $\delta \mathbf{r}_{i}\left(q_{i}\right)$ and $\delta \boldsymbol{\theta}_{i}\left(q_{i}\right)$ are the movement error vectors along and about the axes of $R_{i}$ and $q_{i}$ is the linear or angular displacement of the $i$ th joint. The elements of $\delta \mathbf{r}_{i}\left(q_{i}\right)$ and $\delta \boldsymbol{\theta}_{i}\left(q_{i}\right)$ have been listed in Table 1 . 
Now, by taking similar account of all location errors and joint motion errors within every joint of the kinematic chain, the actual workpiece pose measured in $R$ is then expressed by

$$
{ }^{0} \mathbf{T}_{W}\left(\mathbf{I}_{4}+{ }^{W} \widehat{\boldsymbol{\Delta}}_{W}\right)=\left(\prod_{i=1}^{4}\left(\mathbf{I}_{4}+\widehat{\boldsymbol{\Theta}}_{i}\right){ }^{i-1} \mathbf{T}_{i}\left(\mathbf{I}_{4}+\widehat{\boldsymbol{\Delta}}_{i}\right)\right){ }^{4} \mathbf{T}_{W}
$$

Expanding (6), ignoring higher-order terms in the errors, and applying appropriate adjoint transformations lead to a vector representation of the linearized pose error evaluated in $R_{W}$. Consider

$$
\begin{aligned}
& { }^{W} \widehat{\boldsymbol{\Delta}}_{W}=\sum_{i=1}^{4}{ }^{W} \mathbf{A}_{i} \boldsymbol{\Delta}_{i}+\sum_{i=1}^{4}{ }^{W} \mathbf{A}_{i-1}{ }^{i-1} \mathbf{P}_{i} \boldsymbol{\Theta}_{i}, \\
& \left.{ }^{W} \mathbf{A}_{i}=\left[\begin{array}{cc}
{ }^{W} \mathbf{R}_{i} & {\left[{ }^{W} \mathbf{r}_{i} \times\right.} \\
\mathbf{0} & { }^{W} \mathbf{R}_{i}
\end{array}\right], \quad{ }^{W} \mathbf{R}_{i}\right] \mathbf{P}_{i}=\left[\begin{array}{cc}
\mathbf{I}_{3} & {\left[{ }^{i-1} \mathbf{r}_{i} \times\right]} \\
\mathbf{0} & \mathbf{I}_{3}
\end{array}\right],
\end{aligned}
$$

where ${ }^{W} \mathbf{A}_{i}$ is the $6 \times 6$ adjoint transformation matrix of $R_{i}$ relative to $R_{W}$ and ${ }^{W} \Delta_{W}, \Delta_{i}$, and $\boldsymbol{\Theta}_{i}$, obtained via identification mapping [21], are the $6 \times 1$ vector representations of ${ }^{W} \widehat{\Delta}_{W}, \widehat{\Delta}_{i}$, and $\widehat{\boldsymbol{\Theta}}_{i}$, respectively.

Finally, premultiplying both sides of (7) with ${ }^{R^{\prime}} \mathbf{A}_{W}$ results in the pose error twist of the workpiece evaluated in $R^{\prime}$ :

$$
\boldsymbol{\$}_{W}={ }^{R^{\prime}} \mathbf{A}_{W}{ }^{W} \boldsymbol{\Delta}_{W}=\sum_{i=1}^{4}{ }^{R^{\prime}} \mathbf{A}_{i} \boldsymbol{\Delta}_{i}+\sum_{i=1}^{4}{ }^{R^{\prime}} \mathbf{A}_{i-1}{ }^{i-1} \mathbf{P}_{i} \boldsymbol{\Theta}_{i},
$$

where ${ }^{R^{\prime}} \mathbf{A}_{i}={ }^{R^{\prime}} \mathbf{A}_{W}{ }^{W} \mathbf{A}_{i}$ is the adjoint transformation matrix of $R_{i}$ relative to $R^{\prime}$.

3.2. Error Modeling for Cutting Tool Kinematic Chain. Figure 2(b) shows the cutting tool kinematic chain of the fiveaxis machine tool, and this chain has only one joint. Similarly, global reference frame $R^{\prime}$ is located at point $O^{\prime}$ on the tool tip. The pose error twist of the tool evaluated in $R^{\prime}$ can be expressed as

$$
\mathbf{\$}_{T}={ }^{R^{\prime}} \mathbf{A}_{i} \Delta_{i}+{ }^{R^{\prime}} \mathbf{A}_{j-1}{ }^{j-1} \mathbf{P}_{j} \boldsymbol{\Theta}_{j},
$$

where $j=1$ and denotes the $Z$-axis.

3.3. Error Modeling for Machine Tool. For the two kinematic chains, since the reference point of the tool and workpiece is the same one and the pose error twists of the tool and workpiece are both evaluated in $R^{\prime}$, the pose error twist of machine tool can be written as

$$
\begin{aligned}
\mathbf{\$}=\mathbf{\$}_{T}-\mathbf{\$}_{W}= & \sum_{i=1}^{4}{ }^{R^{\prime}} \mathbf{A}_{i} \boldsymbol{\Delta}_{i}-{ }^{R^{\prime}} \mathbf{A}_{j} \boldsymbol{\Delta}_{j} \\
& +\sum_{i=1}^{4}{ }^{R^{\prime}} \mathbf{A}_{i-1}{ }^{i-1} \mathbf{P}_{i} \boldsymbol{\Theta}_{i}-{ }^{R^{\prime}} \mathbf{A}_{j-1}{ }^{j-1} \mathbf{P}_{j} \boldsymbol{\Theta}_{j}
\end{aligned}
$$

In (10), the pose error twist of the machine tool is represented as the linear combination of the two kinds of geometric source errors, joint motion errors, and location errors. Therefore, the mapping from each source error to the pose accuracy can directly be obtained with explicit physical meaning. \$ can also be written in matrix form:

$$
\mathbf{\$}=\mathbf{M} \boldsymbol{\varepsilon}=\left[\begin{array}{ll}
\mathbf{M}_{T} & -\mathbf{M}_{W}
\end{array}\right]\left(\begin{array}{c}
\boldsymbol{\varepsilon}_{T} \\
\boldsymbol{\varepsilon}_{W}
\end{array}\right),
$$

where

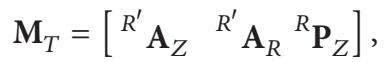

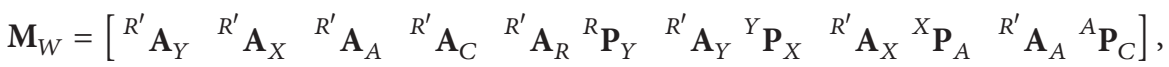

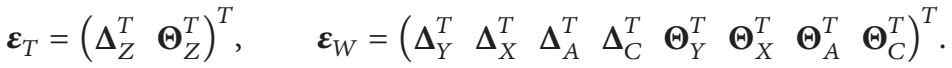

In (11), all geometric source errors are written in the vector form as follows:

$$
\begin{gathered}
\Delta_{Z}=\left(\begin{array}{llllll}
\delta_{x}(z) & \delta_{y}(z) & \delta_{z}(z) & \varepsilon_{x}(z) & \varepsilon_{y}(z) & \varepsilon_{z}(z)
\end{array}\right)^{T}, \\
\boldsymbol{\Theta}_{Z}=\left(\begin{array}{llllll}
0 & 0 & 0 & S_{z y} & S_{z x} & 0
\end{array}\right)^{T} \\
\Delta_{Y}=\left(\begin{array}{llllll}
\delta_{x}(y) & \delta_{y}(y) & \delta_{z}(y) & \varepsilon_{x}(y) & \varepsilon_{y}(y) & \varepsilon_{z}(y)
\end{array}\right)^{T}, \\
\boldsymbol{\Theta}_{Y}=\mathbf{0}_{6 \times 1} \\
\Delta_{X}=\left(\begin{array}{llllll}
\delta_{x}(x) & \delta_{y}(x) & \delta_{z}(x) & \varepsilon_{x}(x) & \varepsilon_{y}(x) & \varepsilon_{z}(x)
\end{array}\right)^{T}, \\
\boldsymbol{\Theta}_{X}=\left(\begin{array}{llllll}
0 & 0 & 0 & 0 & 0 & S_{x y}
\end{array}\right)^{T},
\end{gathered}
$$

$$
\begin{gathered}
\Delta_{A}=\left(\begin{array}{llllll}
\delta_{x}(\alpha) & \delta_{y}(\alpha) & \delta_{z}(\alpha) & \varepsilon_{x}(\alpha) & \varepsilon_{y}(\alpha) & \varepsilon_{z}(\alpha)
\end{array}\right)^{T}, \\
\Theta_{A}=\left(\begin{array}{llllll}
\delta_{x A} & \delta_{y A} & \delta_{z A} & 0 & S_{\alpha z} & S_{\alpha y}
\end{array}\right)^{T} \\
\Delta_{C}=\left(\begin{array}{lllllll}
\delta_{x}(\gamma) & \delta_{y}(\gamma) & \delta_{z}(\gamma) & \varepsilon_{x}(\gamma) & \varepsilon_{y}(\gamma) & \varepsilon_{z}(\gamma)
\end{array}\right)^{T}, \\
\Theta_{C}=\left(\begin{array}{lllllll}
0 & \delta_{y C} & 0 & S_{\gamma y} & S_{\gamma x} & 0
\end{array}\right)^{T} .
\end{gathered}
$$

Equation (11) can also be written in the following form:

$$
\mathbf{\$}=\left(\begin{array}{c}
\Delta \mathbf{r} \\
\Delta \boldsymbol{\theta}
\end{array}\right)=\left[\begin{array}{l}
\mathbf{M}_{r} \\
\mathbf{M}_{\theta}
\end{array}\right] \boldsymbol{\varepsilon}=\mathbf{M} \boldsymbol{\varepsilon},
$$


where $\Delta \mathbf{r}=\left(\begin{array}{lll}\Delta x & \Delta y & \Delta z\end{array}\right)^{T}$ and $\Delta \boldsymbol{\theta}=\left(\begin{array}{lll}\Delta \alpha & \Delta \beta & \Delta \gamma\end{array}\right)^{T}$ are the positional and orientational error vectors measured in $R^{\prime}$, respectively.

\section{Error Separation}

Theoretically, the effects of source errors on pose accuracy of 5-axis machine tools cannot fully be compensated by software, and only those pose errors corresponding to the permissible motion types are compensable by means of error compensation. Therefore, it is essential to distinguish the source errors affecting uncompensable pose errors from those affecting compensable ones. Only then can suitable measures be adopted for accuracy improvement via component tolerance design, manufacturing, and assembly, as well as error compensation.

In order to identify the uncompensable source errors from $\varepsilon$, we should first ascertain the directions of uncompensable pose error of machine tool at any configuration. From the view of motion and constrain, uncompensable directions are the constrained directions of machine tool system. Since they are not parallel to any actuated joint's axis, in other words, the actuating forces or torques of machine tool cannot do work in these uncompensable directions, the uncompensable pose errors cannot be compensated by modifying the ideal output of actuated joints.

Generally, the position errors of cutting tool with respect to workpiece can be compensated by the three mutually perpendicular translational axes. Therefore, only the orientational errors need to be analyzed. Figure 3 shows the direction of uncompensable orientational error of the 5-axis machine tool (only the tilting rotary table is drawn). $\mathbf{a}$ and $\mathbf{c}$ denote the unit vectors of rotary axes $A$ and $C$, and $\mathbf{n}=\mathbf{c} \times \mathbf{a}$. Obviously, the orientational error about direction $\mathbf{n}$ is uncompensable.

According to (14), we have

$$
\begin{aligned}
\Delta \mathbf{r} & =\mathbf{M}_{r} \boldsymbol{\varepsilon}, \\
\Delta \boldsymbol{\theta} & =\mathbf{M}_{\theta} \boldsymbol{\varepsilon} .
\end{aligned}
$$

Taking dot products on both sides of (16) with uncompensable direction vector $\mathbf{n}$ leads to

$$
\mathbf{n}^{T} \Delta \boldsymbol{\theta}=\mathbf{n}^{T} \mathbf{M}_{\theta} \boldsymbol{\varepsilon}=\sum_{j=1}^{n} a_{j} \varepsilon_{j}, \quad a_{j}=\sum_{k=1}^{3} n_{k} M_{\theta, k j},
$$

where $\varepsilon_{j}$ is the $j$ th component in $\boldsymbol{\varepsilon}, a_{j}$ is the coefficient of $\varepsilon_{i}, n$ is the number of geometric source errors in $\boldsymbol{\varepsilon}, n_{k}$ is the $k$ th component in $\mathbf{n}$, and $M_{\theta, k j}$ is the element of row $k$ and column $j$ in $\mathbf{M}_{\theta}$. The geometric source error $\varepsilon_{j}$ whose coefficient $a_{j} \neq 0$ will affect the uncompensable orientational error about direction $\mathbf{n}$.

Let $\Delta \boldsymbol{\theta}^{\prime}=\left(\begin{array}{lll}\Delta \alpha^{\prime} & \Delta \beta^{\prime} & \Delta \gamma^{\prime}\end{array}\right)=\mathbf{n}^{T} \Delta \boldsymbol{\theta} . \Delta \alpha^{\prime}, \Delta \beta^{\prime}$, and $\Delta \gamma^{\prime}$ are the orientational errors about $\mathbf{a}, \mathbf{n}$, and $\mathbf{c}$, respectively. With the method mentioned above, all the uncompensable source errors can be identified. Thus, (14) can be rewritten in terms of the compensable and uncompensable degrees of freedom such that

$$
\begin{gathered}
\Delta \mathbf{p}_{c}=\left(\begin{array}{cccc}
\Delta x & \Delta y & \Delta z & \Delta \alpha^{\prime} \quad \Delta \gamma^{\prime}
\end{array}\right)^{T}=\mathbf{M}_{c} \boldsymbol{\varepsilon}_{c}, \\
\Delta \mathbf{p}_{u}=\left(\Delta \beta^{\prime}\right)=\mathbf{M}_{u} \mathbf{e}_{u},
\end{gathered}
$$

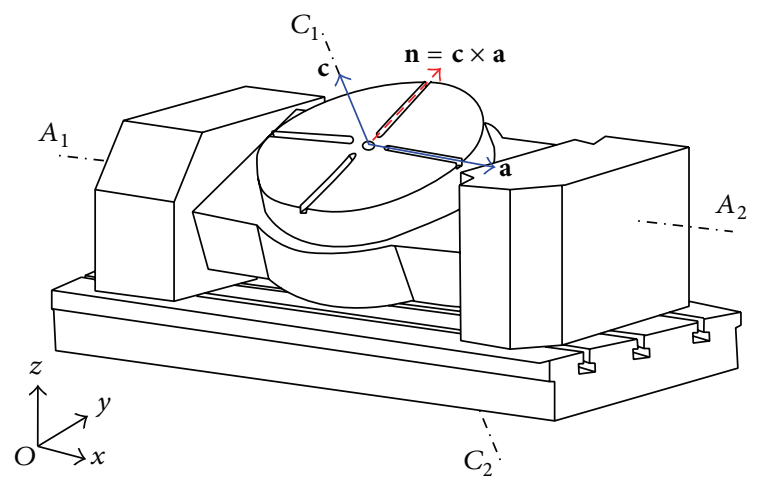

$\longrightarrow$ Directions of compensable orientational errors (direction of actuated torque)

$-\rightarrow$ Direction of uncompensable orientational error (directions of constrained torques)

-.-. Axes of actuated rotational joints

FIGURE 3: Direction of uncompensable orientational error of 5-axis machine tool.

where $\Delta \mathbf{p}_{c}$ denotes the 5-dimensional compensable pose error vector, $\Delta \mathbf{p}_{u}$ denotes the 1-dimensional uncompensable pose error vector, and $\boldsymbol{\varepsilon}_{c}\left(\boldsymbol{\varepsilon}_{u}\right)$ denotes the compensable (uncompensable) geometric source error vector. For the 5axis machine tool analyzed in this paper, $\boldsymbol{\varepsilon}_{c}=\boldsymbol{\varepsilon}$, and

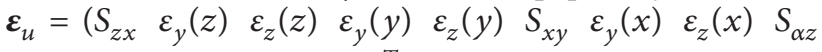
$\left.S_{\alpha y} \varepsilon_{y}(\alpha) S_{\gamma x} \varepsilon_{x}(\gamma) \varepsilon_{y}(\gamma)\right)^{T}$, which should be eliminated or at least minimized during the manufacturing and assembly processes.

\section{Sensitivity Analysis}

After the error model is established and the compensable/uncompensable source errors are separated, a probability global sensitivity model is proposed. And, then, the sensitivities of compensable/uncompensable pose accuracies of the machine tool with respect to geometric source errors are analyzed, respectively.

5.1. Probability Model. In order to investigate the influences of the geometric errors on the volumetric accuracy in a statistical sense, a probability model is needed. Taking the norm on both sides of (14) yields

$$
(\$)^{2}=\$^{T} \$=\boldsymbol{\varepsilon}^{T} \mathbf{M}^{T} \mathbf{M} \boldsymbol{\varepsilon}=\sum_{i=1}^{3}\left(\sum_{j=1}^{n} M_{i j} \varepsilon_{j}\right)^{2},
$$

where $\varepsilon_{j}$ is the $j$ th element in $\boldsymbol{\varepsilon}, M_{i j}$ is the element of row $i$ and column $j$ in $\mathbf{M}$, and $n$ is the number of geometric errors. Assume that all elements in $\varepsilon$ are independent and of zero mean. This allows the mean value of $\$$ to be zero; that is, $E$ $(\$)=0$, where $E$ is the statistical expectation. As a result, the variance of $\$$ can be expressed by

$$
E\left(\$^{2}\right)=\sum_{i=1}^{3}\left(\sum_{j=1}^{n} M_{i j}^{2}\right) E\left(\varepsilon_{j}^{2}\right) .
$$


Thus, the standard deviation of $\$$ can be represented by

$$
\sigma(\$)=\sqrt{\sum_{j=1}^{n} \mu_{j}^{2} D\left(\varepsilon_{j}\right)}=\sqrt{\sum_{j=1}^{n} \mu_{j}^{2} \sigma^{2}\left(\varepsilon_{j}\right)},
$$

where $\mu_{j}=\sqrt{\sum_{i=1}^{3} M_{i j}^{2}}$ is defined as the volumetric sensitivity of $\$$ with respect to $\varepsilon_{j}$, representing the standard deviation of $\$$ caused by the unit standard deviation of $\varepsilon_{j}$. Note that $\mu_{j}$ varies with the configuration of the machine tool; therefore, its mean value can be employed as an index to evaluate the influence of $\varepsilon_{j}$ on the pose accuracy in the overall workspace. This index is defined as

$$
\bar{\mu}_{j}=\frac{\left(\int_{V} \mu_{j} d V\right)}{V}
$$

where $V$ is the volume of the prescribed workspace.

According to the sensitivity analysis model proposed above, the sensitivities of the elements of $\Delta \mathbf{p}_{c}\left(\Delta \mathbf{p}_{u}\right)$ with respect to the elements of $\boldsymbol{\varepsilon}_{c}\left(\boldsymbol{\varepsilon}_{u}\right)$ can be expressed as

$$
\begin{gathered}
\bar{\mu}_{x, j}=\frac{\left(\int_{V}\left|\mathbf{M}_{c, 1, j}\right| d V\right)}{V} \\
\bar{\mu}_{y, j}=\frac{\left(\int_{V}\left|\mathbf{M}_{c, 2, j}\right| d V\right)}{V} \\
\bar{\mu}_{z, j}=\frac{\left(\int_{V}\left|\mathbf{M}_{c, 3, j}\right| d V\right)}{V} \\
\bar{\mu}_{\alpha, j}=\frac{\left(\int_{V}\left|\mathbf{M}_{c, 4, j}\right| d V\right)}{V} \\
\bar{\mu}_{\gamma, j}=\frac{\left(\int_{V}\left|\mathbf{M}_{c, 5, j}\right| d V\right)}{V}, \\
\bar{\mu}_{\beta, j}=\frac{\left(\int_{V}\left|\mathbf{M}_{u, 1, j}\right| d V\right)}{V \quad j=1,2, \ldots, 14 .}
\end{gathered}
$$

5.2. Sensitivity Analysis. Utilizing the indices given by (23) and (24), the sensitivity analysis is carried out for the machine tool, whose prescribed workspace is listed in Table 2 . The structure parameters are shown in Table 3. It should be noted that the parameter vector ${ }^{C} \mathbf{r}_{W}$, the position vector of cutting point measured in frame $C$, changes with different machine configuration. Therefore, it should be calculated at every given configuration. Table 4 shows the numbers for corresponding error components [20].

In the processes of sensitivity analysis, all error components are considered as the same value, that is, $1 \mu \mathrm{m}$ for position errors and $1 \mu \mathrm{m} / \mathrm{m}$ for angular errors [20]. The sensitivity analysis results of (23) and (24) are shown in Figures 4 and 5, respectively. It can be found that linear source errors only have influences on position errors of the tool relative to the workpiece, while angular source errors having influences on both position and orientation errors. In
TABLE 2: Workspace of the machine tool.

\begin{tabular}{lcccc}
\hline$X(\mathrm{~mm})$ & $Y(\mathrm{~mm})$ & $Z(\mathrm{~mm})$ & $A(\mathrm{rad})$ & $B(\mathrm{rad})$ \\
\hline $0 \sim 600$ & $0 \sim 400$ & $0 \sim 400$ & $-\pi / 2 \sim \pi / 2$ & $0 \sim 2 \pi$ \\
\hline
\end{tabular}

TABLE 3: Structure parameters of the machine tool.

\begin{tabular}{lc}
\hline Structure parameters & Values $(\mathrm{mm})$ \\
\hline$r_{X x}, r_{Y x}, r_{Z x}, r_{A x}, r_{C x}, r_{T x}$ & $300,-400,-400,0,0,0$ \\
$r_{X y}, r_{Y y}, r_{Z y}, r_{A y}, r_{C y}, r_{T y}$ & $0,0,1000,0,0,-400$ \\
$r_{X z}, r_{Y z}, r_{Z z}, r_{A z}, r_{C z}, r_{T z}$ & $200,600,1200,400,0,-500$ \\
\hline
\end{tabular}

TABLE 4: Number of error components.

\begin{tabular}{lc}
\hline Number & Error components \\
\hline $1,2,3,4,5,6$ & $\delta_{x}(x), \delta_{y}(x), \delta_{z}(x), \varepsilon_{x}(x), \varepsilon_{y}(x), \varepsilon_{z}(x)$ \\
$7,8,9,10,11,12$ & $\delta_{x}(y), \delta_{y}(y), \delta_{z}(y), \varepsilon_{x}(y), \varepsilon_{y}(y), \varepsilon_{z}(y)$ \\
$13,14,15,16,17,18$ & $\delta_{x}(z), \delta_{y}(z), \delta_{z}(z), \varepsilon_{x}(z), \varepsilon_{y}(z), \varepsilon_{z}(z)$ \\
$19,20,21,22,23,24$ & $\delta_{x}(\alpha), \delta_{y}(\alpha), \delta_{z}(\alpha), \varepsilon_{x}(\alpha), \varepsilon_{y}(\alpha), \varepsilon_{z}(\alpha)$ \\
$25,26,27,28,29,30$ & $\delta_{x}(\gamma), \delta_{y}(\gamma), \delta_{z}(\gamma), \varepsilon_{x}(\gamma), \varepsilon_{y}(\gamma), \varepsilon_{z}(\gamma)$ \\
$31,32,33,34,35,36,37$ & $S_{z y}, S_{z x}, S_{x y}, S_{\alpha z}, S_{\alpha y}, S_{\gamma y}, S_{\gamma x}$ \\
$38,39,40,41$ & $\delta_{x A}, \delta_{y A}, \delta_{z A}, \delta_{y C}$ \\
\hline
\end{tabular}

Figure 4, we can easily find the key error components for each compensable direction. For example, the error components 5 , $6,11,12,24,30,32,33,34$, and 35 have more influences on the orientational error $\Delta \gamma$ than other error components. Figure 5 shows the sensitivity of uncompensable orientational error $\Delta \beta$ with respect to every error component in $\boldsymbol{\varepsilon}_{u}$. The sensitivities of $\varepsilon_{y}(\alpha)$ and $S_{\gamma x}$ are 1 , since the direction of $\varepsilon_{y}(\alpha)$ and $S_{\gamma x}$ always coincides with the uncompensable direction n. The other components in $\boldsymbol{\varepsilon}_{u}$ have similar value of $0.6 \sim$ 0.7. The above sensitivity analysis results can be used for the accuracy design of the machine tool, and the key error components should be paid more attention to in manufacture and assembly processes.

\section{Conclusions}

The proposed error modeling methodology in this paper allows the geometric source errors affecting the compensable and uncompensable pose accuracy of the five-axis machine tool to be identified in an explicit manner. The merit of this approach is that it can provide designers and/or field engineers with an informative guideline to adopt suitable measures for improving accuracy. The model has clear physical meanings and shows higher computational efficiency in numerical simulations without considering the nonlinear effects.

The sensitivities of compensable and uncompensable pose accuracies have been analyzed, which will be helpful in the accuracy design of a machine tool. It will enhance the understanding of which error components are more important or influence the machining target, and these error components should be paid more consideration to in design processes. 


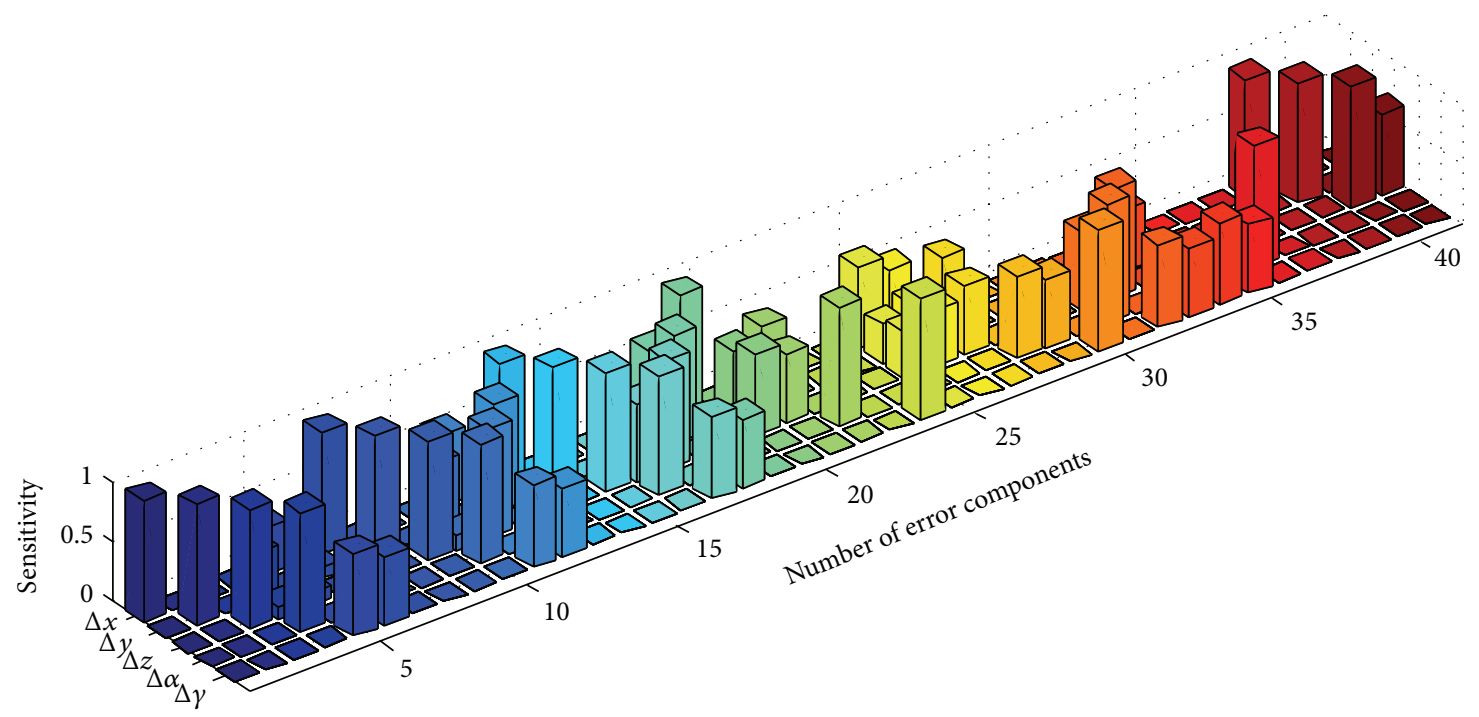

FIGURE 4: Sensitivities of $\Delta \mathbf{p}_{c}$ with respect to $\boldsymbol{\varepsilon}_{c}$.

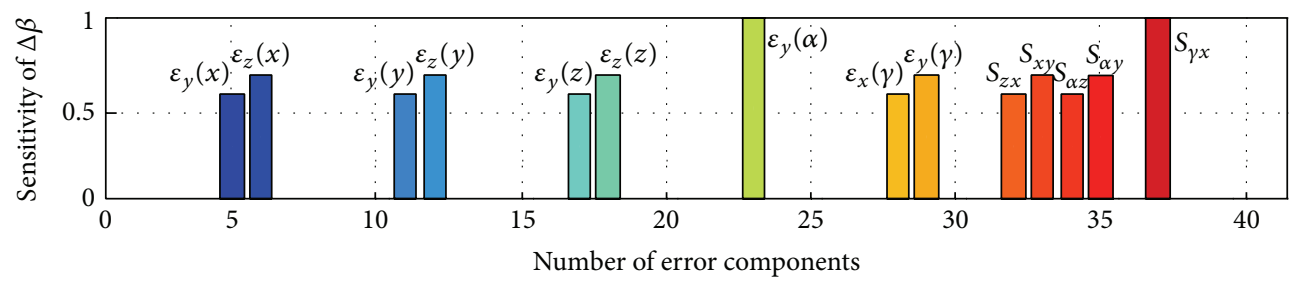

Figure 5: Sensitivities of $\Delta \mathbf{p}_{u}$ with respect to $\boldsymbol{\varepsilon}_{u}$.

\section{Conflict of Interests}

The authors declare that there is no conflict of interests regarding the publication of this paper.

\section{Acknowledgment}

This work was supported by a Grant from the National High Technology Research and Development Program of China (no. 2012AA040701).

\section{References}

[1] R. Ramesh, M. A. Mannan, and A. N. Poo, "Error compensation in machine tools-a review. Part I. Geometric, cutting-force induced and fixture-dependent errors," International Journal of Machine Tools and Manufacture, vol. 40, no. 9, pp. 1235-1256, 2000.

[2] H. Schwenke, W. Knapp, H. Haitjema, A. Weckenmann, R. Schmitt, and F. Delbressine, "Geometric error measurement and compensation of machines-An update," CIRP AnnalsManufacturing Technology, vol. 57, no. 2, pp. 660-675, 2008.

[3] W. Tian, W. Gao, D. Zhang et al., "A general approach for error modeling of machine tools," International Journal of Machine Tools and Manufacture, vol. 79, pp. 17-23, 2014.

[4] V. Kiridena and P. M. Ferreira, "Mapping the effects of positioning errors on the volumetric accuracy of five-axis $\mathrm{CNC}$ machine tools," International Journal of Machine Tools and Manufacture, vol. 33, no. 3, pp. 417-437, 1993.

[5] A. K. Srivastava, S. C. Veldhuis, and M. A. Elbestawit, "Modelling geometric and thermal errors in a five-axis cnc machine tool," International Journal of Machine Tools and Manufacture, vol. 35, no. 9, pp. 1321-1337, 1995.

[6] B. K. Jha and A. Kumar, "Analysis of geometric errors associated with five-axis machining centre in improving the quality of cam profile," International Journal of Machine Tools and Manufacture, vol. 43, no. 6, pp. 629-636, 2003.

[7] Y. Lin and Y. Shen, "Modelling of five-axis machine tool metrology models using the matrix summation approach," International Journal of Advanced Manufacturing Technology, vol. 21, no. 4, pp. 243-248, 2003.

[8] S. H. Yang, Real-time compensation for geometric, thermal, and cutting force induced errors in machining tools [Ph.D. thesis], University of Michigan, Ann Arbor, Mich, USA, 1996.

[9] Y. Y. Hsu and S. S. Wang, "A new compensation method for geometry errors of five-axis machine tools," International Journal of Machine Tools and Manufacture, vol. 47, no. 2, pp. 352360, 2007.

[10] J. H. Lee, Y. Liu, and S. H. Yang, "Accuracy improvement of miniaturized machine tool: Geometric error modeling and compensation," International Journal of Machine Tools and Manufacture, vol. 46, no. 12-13, pp. 1508-1516, 2006.

[11] K. G. Ahn and D. W. Cho, "Analysis of the volumetric error uncertainty of a three-axis machine tool by beta distribution," 
International Journal of Machine Tools and Manufacture, vol. 40, no. 15, pp. 2235-2248, 2000.

[12] M. Rahman, J. Heikkala, and K. Lappalainen, "Modeling, measurement and error compensation of multi-axis machine tools. Part I. Theory," International Journal of Machine Tools and Manufacture, vol. 40, no. 10, pp. 1535-1546, 2000.

[13] A. C. Okafor and Y. M. Ertekin, "Derivation of machine tool error models and error compensation procedure for three axes vertical machining center using rigid body kinematics," International Journal of Machine Tools and Manufacture, vol. 40, no. 8, pp. 1199-1213, 2000.

[14] S. Zhu, G. Ding, S. Qin, J. Lei, L. Zhuang, and K. Yan, "Integrated geometric error modeling, identification and compensation of CNC machine tools," International Journal of Machine Tools and Manufacture, vol. 52, no. 1, pp. 24-29, 2012.

[15] T. Huang, D. J. Whitehouse, and D. G. Chetwynd, "A unified error model for tolerance design, assembly and error compensation of 3-DOF parallel kinematic machines with parallelogram struts," CIRP Annals-Manufacturing Technology, vol. 51, no. 1, pp. 297-301, 2002.

[16] H. Chanal, F. Paccot, and E. Duc, "Sensitivity analysis of an overconstrained parallel structure machine tool, the Tripteor X7," Applied Mechanics and Materials, vol. 162, pp. 394-402, 2012.

[17] K. C. Fan, H. Wang, J. W. Zhao, and T. H. Chang, "Sensitivity analysis of the 3-PRS parallel kinematic spindle platform of a serial-parallel machine tool," International Journal of Machine Tools and Manufacture, vol. 43, no. 15, pp. 1561-1569, 2003.

[18] S. M. Wang and J. J. Lin, "On-machine volumetric-error measurement and compensation methods for micro machine tools," International Journal of Precision Engineering and Manufacturing, vol. 14, no. 6, pp. 989-994, 2013.

[19] A. J. Patel and K. F. Ehmann, "Volumetric error analysis of a Stewart platform-based machine tool," CIRP Annals-Manufacturing Technology, vol. 46, no. 1, pp. 287-290, 1997.

[20] G. Chen, Y. Liang, Y. Sun et al., "Volumetric error modeling and sensitivity analysis for designing a five-axis ultra-precision machine tool," The International Journal of Advanced Manufacturing Technology, vol. 68, no. 9-12, pp. 2525-2534, 2013.

[21] L. W. Tsai, Robot Analysis: The Mechanics of Serial and Parallel Manipulators, John Wiley \& Sons, New York, NY, USA, 1999. 


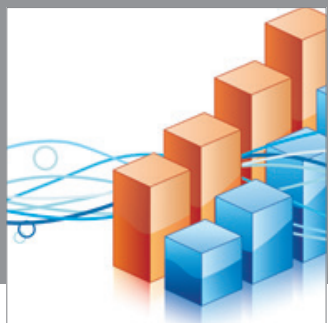

Advances in

Operations Research

mansans

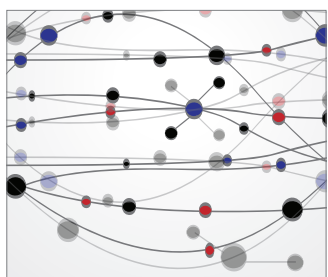

The Scientific World Journal
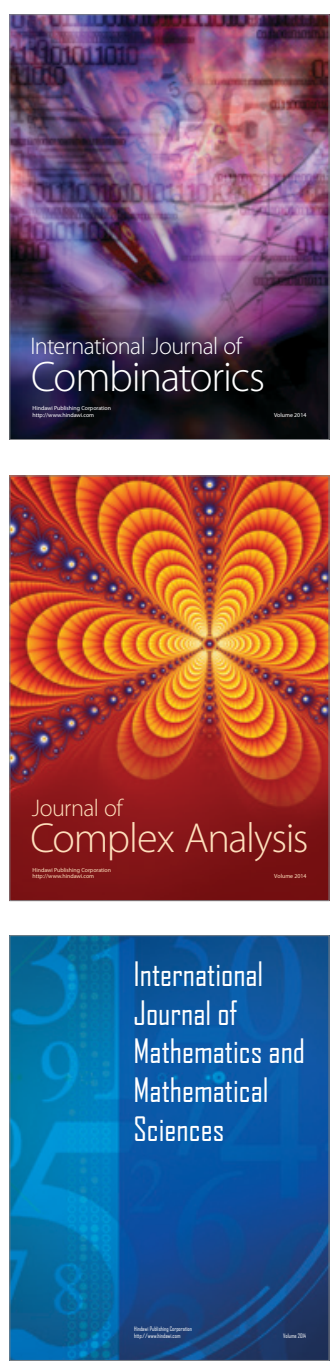
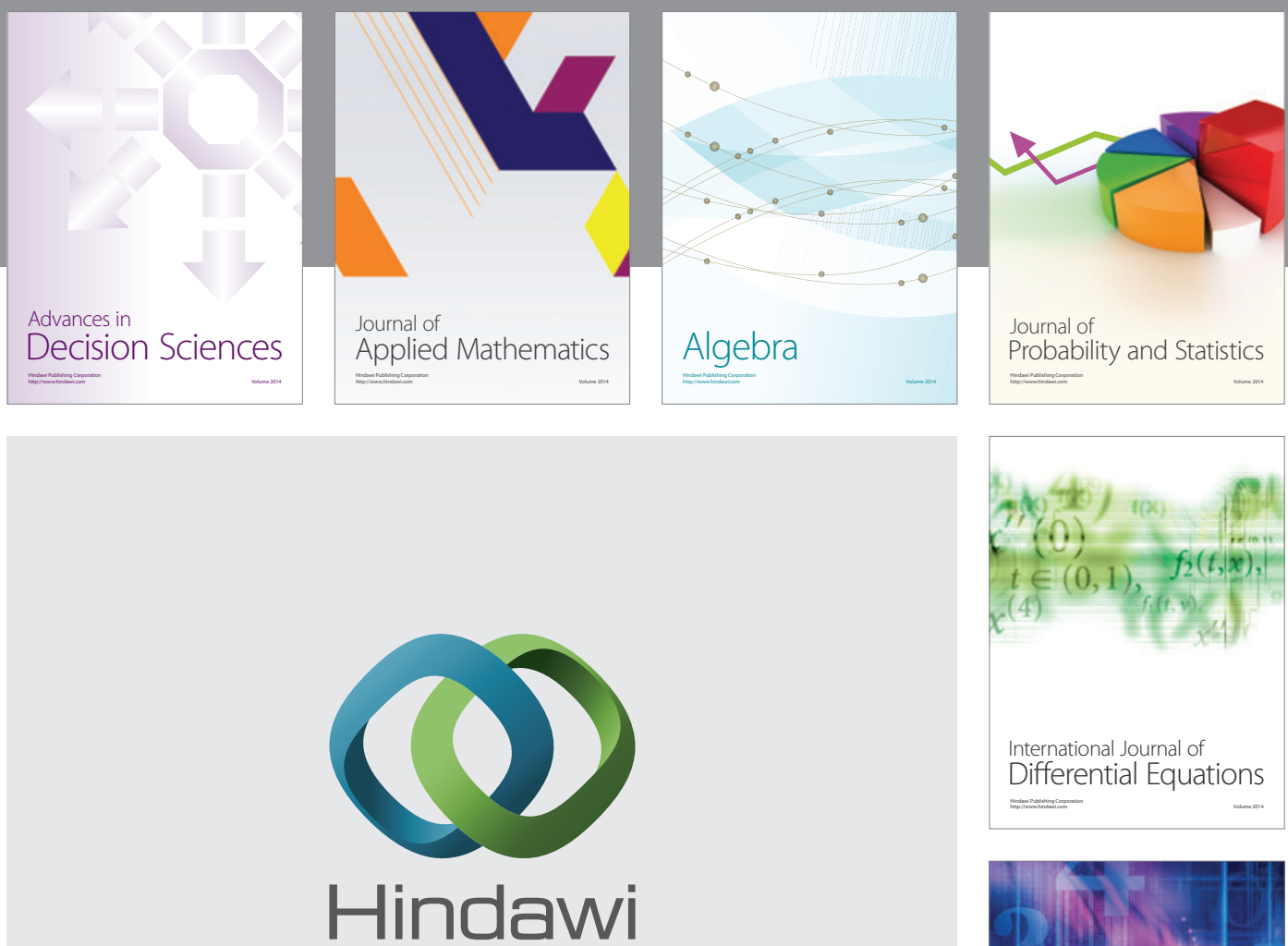

Submit your manuscripts at http://www.hindawi.com
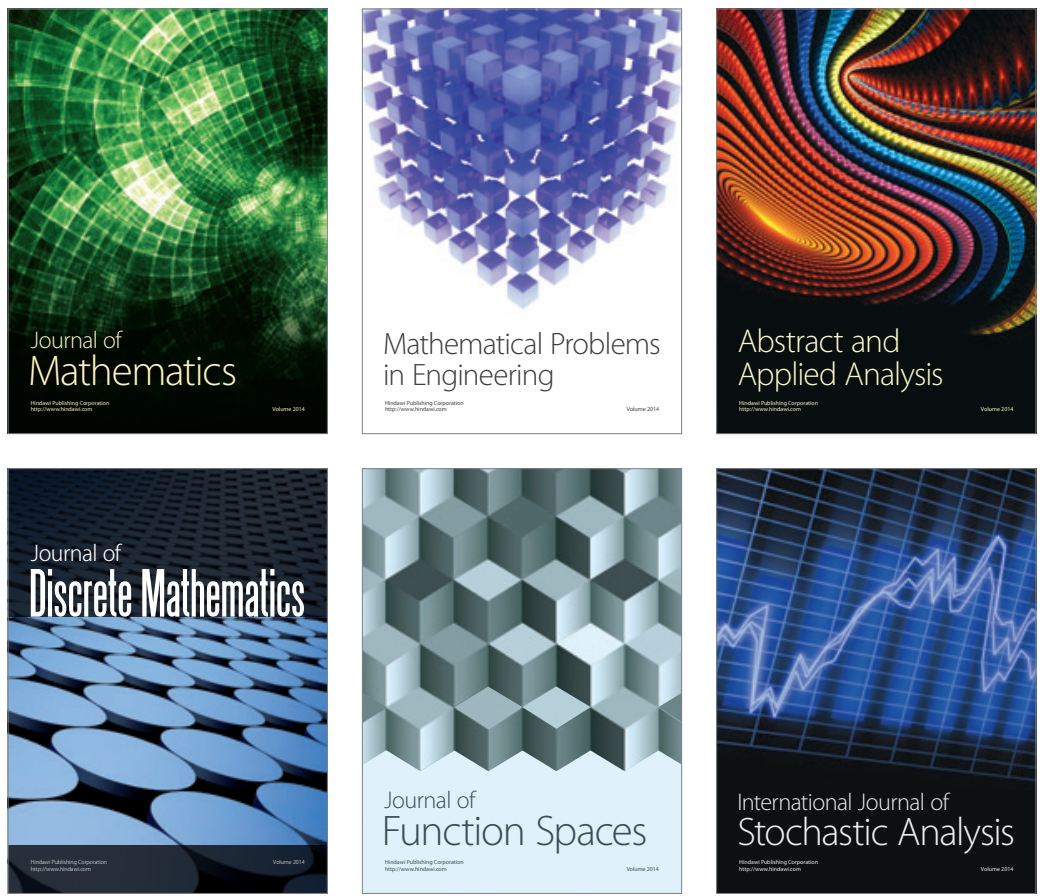

Journal of

Function Spaces

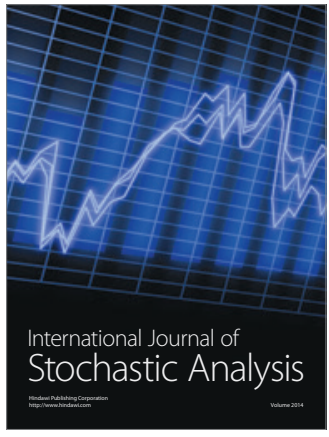

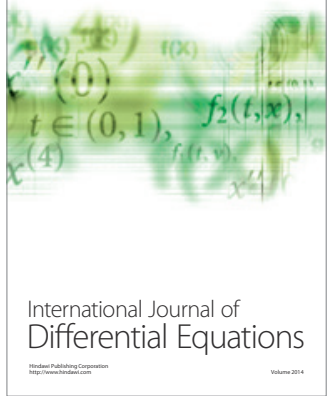
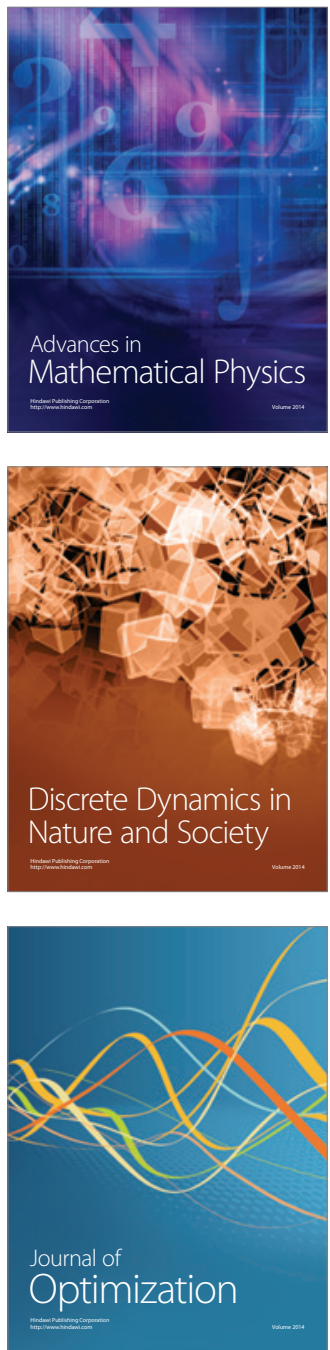